\title{
Phonon-Assisted Kondo Resonance in Spin-Dependent Transport through a Quantum Dot
}

\author{
R. ŚWIRKOWICZ*, M. WILCZYŃSKI \\ Faculty of Physics, Warsaw University of Technology \\ Koszykowa 75, 00-662 Warsaw, Poland \\ AND J. BARNAŚ \\ Department of Physics, Adam Mickiewicz University \\ Umultowska 85, 61-614 Poznań, Poland \\ and Institute of Molecular Physics, Polish Academy of Sciences \\ M. Smoluchowskiego 17, 60-179 Poznań, Poland
}

\begin{abstract}
Effects of local vibrational modes on electron transport through a quantum dot attached to ferromagnetic electrodes are studied in the Kondo regime by the non-equilibrium Green function formalism based on the equation of motion method. Differential conductance is calculated for parallel and antiparallel configurations of the leads' magnetic moments, and well defined Kondo resonance peaks and their phonon satellites are found. The influence of a compensating magnetic field on the peak positions is also discussed.
\end{abstract}

PACS numbers: 73.23.Hk, 73.63.Kv, 73.43.Qt, 72.25.-b

\section{Introduction}

Recent experimental data on electron transport through molecules and quantum dots (QDs) have revealed features which indicate on the role of vibrational degrees of freedom [1]. In particular, the latest experiments have revealed phonon satellites in the Kondo regime due to enhanced electron-phonon coupling in the molecule [2]. These observations have stimulated great interest in theoretical studies of the interplay between electron-phonon interaction (EPI) and the Kondo effect [3]. Spin polarization of conduction electrons in ferromagnetic electrodes leads to additional features of the Kondo phenomenon, not observed in the case of nonmagnetic leads. Coupling of the dot to ferromagnetic electrodes gives rise to an effective exchange field $B_{\text {ex }}$ acting on the dot, which leads to renormalization and splitting of the relevant energy level [4-6]. As a result the conductance zero-bias Kondo anomaly becomes split and considerably suppressed. Spin-polarized transport through a QD coupled to ferromagnetic electrodes with non-collinear magnetic moments in a presence of electron-phonon interaction in the dot was a subject of our previous paper [6] and variation of the main Kondo peaks and phonon satellites with angle between magnetic moments of the leads was analyzed. In the present paper we study phonon assisted transport in the Kondo regime and discuss the interplay between the effective field $B_{\text {ex }}$ generated by the ferromagnetic electrodes and external

* corresponding author; e-mail: renatas@if.pw.edu.pl magnetic field applied to the system. Phonon-assisted Kondo resonance in junctions with a dot asymmetrically connected to two different ferromagnetic electrodes will be also investigated.

\section{Model}

The system which consists of a QD coupled to a phonon bath and connected to external electrodes is described by the Hamiltonian $H=H_{\mathrm{L}}+H_{\mathrm{R}}+H_{\mathrm{ph}}+H_{\mathrm{D}}+H_{\mathrm{T}}$, where $H_{\beta}(\beta=\mathrm{L}, \mathrm{R})$ represents noninteracting electrons in the left and right electrodes, $H_{\mathrm{ph}}$ describes the local vibrational mode of energy $\omega$, whereas $H_{\mathrm{D}}$ is the dot Hamiltonian which includes a standard Anderson term and an electron-phonon interaction part. Tunnelling processes between QD and ferromagnetic electrodes are described by $H_{\mathrm{T}}$ and are characterized by spin-dependent coupling strengths $\Gamma_{\sigma}^{\beta}=\Gamma_{0}^{\beta}\left(1+\hat{\sigma} p_{\beta}\right)$ with $p_{\beta}$ being the spin polarization factor in the lead $\beta$ and $\hat{\sigma}= \pm 1 . \Gamma_{0}^{\beta}$ is constant within the electron band of width $D$ and zero otherwise.

Electron transport is studied within the non-equilibrium Green function formalism described in detail in Ref. [6]. The electron and phonon subsystems are decoupled by applying the Lang-Firsov transformation with phonon operators replaced by their expectation values $[3,6-8]$. Then, QD is described by the Hamiltonian $\tilde{H}_{\mathrm{D}}$ which is the Anderson-type Hamiltonian, but with the renormalized energy level $\epsilon_{\sigma} \rightarrow \tilde{\epsilon}_{\sigma}$ and the renormalized correlation parameter $U \rightarrow \tilde{U}$. Tunneling couplings with electrodes are also renormalized $\Gamma_{\sigma}^{\beta} \rightarrow \tilde{\Gamma}_{\sigma}^{\beta}$. The 
transformation allows one to determine Green functions $\tilde{G}_{\sigma}$ in the Kondo regime with the use of standard procedures [6]. The splitting of the dot level $\tilde{\epsilon}_{\sigma}$ due to ferromagnetism of external electrodes is taken into account via the effective exchange field $B_{\text {ex }}[4-7]$. This field depends on the renormalized coupling strength $\tilde{\Gamma}_{\sigma}^{\beta}$. The electric current and spectral density are calculated according to standard formulae [9], but with the lesser $G_{\sigma}^{<}$ and greater $G_{\sigma}^{>}$Green functions expressed by the appropriate functions $\tilde{G}_{\sigma}^{<(>)}$determined according to the Keldysh equation with the $\mathrm{Ng}$ ansatz applied [6].

\section{Results and discussion}

Numerical calculations were performed in the limit $U \rightarrow \infty$ for the following values of the parameters (measured in the units of $\tilde{D}=D / 50$ ): bare dot level $\epsilon_{\sigma}=\epsilon_{0}=-0.31$, phonon energy $\omega=0.05, k T=0.001$. The EPI coupling strength was assumed to be equal to $g=0.4$. Differential conductance $G_{\text {diff }}=\mathrm{d} I / \mathrm{d} V$ as a function of the bias voltage is presented in Fig. 1 for two configurations of the leads' moments, namely parallel (P) and antiparallel (AP) ones. For symmetric junctions with $\Gamma_{0}^{\mathrm{L}}=\Gamma_{0}^{\mathrm{R}}=0.1$ and $p_{\mathrm{L}}=p_{\mathrm{R}}=0.4$, the Kondo peak in the AP configuration appears at the zero bias limit (Fig. 1a). This is a consequence of the compensation of


Fig. 1. Differential conductance as a function of bias voltage for symmetric (a) and non-symmetric (b) junctions calculated for parameters indicated in the figures. Other parameters are: $k T=0.001, \omega=0.05$, $\epsilon_{0}=-0.31$.

effective exchange fields from the two leads. In a presence of electron-phonon interaction the intensity of the main Kondo resonance is described by the product of renormalized couplings to both electrodes $\tilde{\Gamma}_{\sigma}^{\mathrm{L}} \tilde{\Gamma}_{\sigma}^{\mathrm{R}}$. It is considerably reduced due to the strong coupling to the phonon bath [6]. Additional suppression of the peak is obtained when the dot is connected to ferromagnetic electrodes with strong polarization factors $p_{\beta}[5]$. Apart from the main resonance well-defined phonon satellites develop. The phonon peaks appear on both sides of the main resonance. The first Kondo satellites, at the energies $\pm \omega$, as well as the second ones appearing at $\pm 2 \omega$ are clearly seen. In the parallel configuration the main Kondo peak becomes split and two components move away from the zero bias limit, one towards positive and the other towards negative bias. The splitting of the resonance is a consequence of the non-zero effective field exerted by the leads on the QD. The Kondo satellites move accordingly and appear at the distance $\omega$ from the main resonance peaks. For assumed parameters the first satellites overlap with the main Kondo peaks and as a result their intensity is considerably enhanced. Position of the main anomalies and their satellites depends on the leads polarization, and the splitting increases with increasing $p$. As an external magnetic field is applied to the system in such a way that it compensates the effective field exerted by ferromagnetic electrodes, the splitting of the main resonance is suppressed. The zero-bias Kondo anomaly is then restored. The Kondo satellites are also shifted and they can be observed at energies $\pm \omega$ (for the first ones) and $\pm 2 \omega$ (for second ones), similarly as in the AP configuration, though intensities of the peaks are different.

Results obtained for a non-symmetric junction with $\Gamma_{0}^{\mathrm{L}}=0.2, \Gamma_{0}^{\mathrm{R}}=0.02$ and $p_{\mathrm{L}}=0.4, p_{\mathrm{R}}=0.8$ are presented in Fig. 1b. In such a case the effective fields exerted on the QD by both ferromagnetic electrodes do not compensate each other even in AP configuration. The Kondo anomaly is then split into two components and the zero bias anomaly does not appear. The differential conductance is then considerably reduced in the limit $V \rightarrow 0$. Moreover, the bias dependence of the conductance is strongly asymmetric. A pronounced Kondo peak as well as its phonon satellites lying at the distances $\omega$ and $2 \omega$ from the main peak can be observed for negative values of $\mathrm{eV}$, whereas the intensity of the components appearing for positive voltages is considerably reduced. Due to a strong asymmetry in the coupling strength and in the leads polarization the channel corresponding to electrons with spin up becomes practically non-conductive in AP configuration as $\Gamma_{\uparrow}^{\mathrm{R}}$ is close to zero. The main contribution to the conductance comes then from electrons with spin down and the left Kondo component whose intensity is mainly determined by the coupling with the left electrode can be well observed, whereas the second component, shifted to the right towards positive bias is strongly reduced. However, in the $\mathrm{P}$ configuration both components of the main Kondo peak as well as their phonon satellites develop for two bias polarizations, though their intensities are different due to asymmetry in the coupling strengths.

Difference in transport properties for $\mathrm{P}$ and AP configurations gives rise to the tunnel magnetoresistance (TMR) effect, which is described by the ratio $\mathrm{TMR}=$ $\left(I_{\mathrm{P}}-I_{\mathrm{AP}}\right) / I_{\mathrm{AP}}$, where $I_{\mathrm{P}}, I_{\mathrm{AP}}$ denote currents flowing in $\mathrm{P}$ and $\mathrm{AP}$ geometries, respectively. Results obtained for symmetric and asymmetric junctions are presented in Fig. 2. Let us note that in the symmetric case TMR changes sign for higher voltages and becomes positive. The effect is due to the presence of EPI. On the other hand, in asymmetric junctions TMR is negative for all 

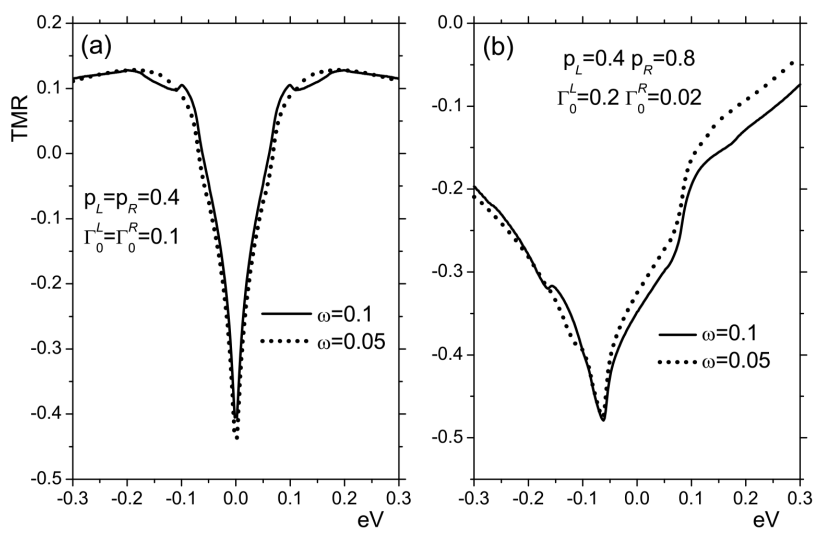

Fig. 2. TMR for symmetric (a) and non-symmetric (b) junctions calculated for parameters indicated in the figures. Other parameters are the same as in Fig. 1.

calculated voltages and is strongly asymmetric with respect to the bias reversal.

\section{Acknowledgments}

This work was supported by funds of the Polish Ministry of Science and Higher Education as a research project in years 2006-2009.

\section{References}

[1] H. Park, J. Park, A.K.L. Lim, E.H. Anderson, A.P. Alivisatos, P.L. McEuen, Nature 407, 57 (2000).

[2] L.H. Yu, Z.K. Keane, J.W. Ciszek, L. Cheng, M.P. Stewart, J.M. Tour, D. Natelson, Phys. Rev. Lett. 93, 266802 (2004); L.H. Yu, D. Natelson, Nano Lett. 4, 79 (2004).

[3] Z.-Z. Chen, R. Lu, B.-F. Zhu, Phys. Rev. B 71, 165324 (2005); M. Galperin, A. Nitzan, M.A. Ratner, Phys. Rev. B 73, 045314 (2006); M. Galperin, A. Nitzan, M.A. Ratner, Phys. Rev. B 76, 035301 (2007).

[4] J. Martinek, M. Sindel, L. Borda, J. Barnaś, J. König, G. Schön, J. von Delft, Phys. Rev. Lett. 91, 247202 (2003); A.N. Pasupathy, R.C. Bialczak, J. Martinek, J.E. Grose, L.A.K. Donev, P.L. McEuen, D.C. Ralph, Science 306, 86 (2004).

[5] R. Świrkowicz, M. Wilczyński, J. Barnaś, J. Phys., Condens. Matter 18, 2291 (2006).

[6] R. Świrkowicz, M. Wilczyński, J. Barnaś, J. Phys., Condens. Matter 20, 255219 (2008).

[7] R.-Q. Wang, Y.-Q. Zhou, D.Y. Xing, J. Phys., Condens. Matter 20, 045219 (2008).

[8] G.D. Mahan, Many-Particle Physics, Plenum Press, New York 2000.

[9] H. Haug, A.-P. Jauho, Quantum Kinetics in Transport and Optics of Semiconductors, Springer-Verlag, Berlin 1996. 